\title{
Multi-Wavelength Radio Features Associated with Large CMEs on Oct. 26-28, 2003
}

\author{
S. J. Wang $\dagger$, Y. Yan, Q. Fu, Y. Liu and Z. Chen \\ National Astronomical Observatories of China, Beijing 100012, China \\ email: wsj@bao.ac.cn
}

\begin{abstract}
We study in focus to the multi-wavelength radio spectra associated with quite strong CME events observed in the time interval of October 26-28, 2003. Using multi-wavelength observations recorded by WIND/WAVES experiment, Learmouth Spectrograph (Australian), PHOENIX-2 (Switzerland), Hiraiso (Japan), DAM/Nançay (France), Izmiran (Russia) and Huairou/NAOC (China), an analyzing of radio bursts was performed for the those events over a large coronal region across the microwave, the decimetric wavelength, the metric wavelength and even to much longer wavelength. The composite spectra indicate there were many complicated structures of radio bursts, including type II bursts, type IV bursts, type III bursts, drifting pulsation structures (DPS) and many radio fine structures (FS).
\end{abstract}

Keywords. Sun: coronal mass ejections (CMEs), radio radiation

\section{Introduction}

There is evidence that many CMEs launch from the low corona and accompanying with rich radio bursts (St. Cyr et al., 1999, Delannèe et al., 2000, Wang et al., 2001), but relatively few imaging data are available to investigate the spatial relationships between bursts and ejections. Therefore a global radio spectra from microwave to metric wavelength across over a large coronal region would be a basic study, and they are essentially needed and useful for the associated faster and/or halo-like CMEs to provide base features and to identify the cause of this association.

\section{Results and Discussions}

In the late 2003, many violent solar activities were observed. We focus to the large CMEs in Oct. 26-28, 2003 which involved large spanning region over more than 140 degree, even up to 360 degree. Also very strong flare activities were recorded in the NOAA 0486 and 0484 during this period with importance of M2.7/2F to X17.2/4B.

An overview of solar radio bursts on 26 and 27 over $1 \mathrm{MHz}-7.6 \mathrm{GHz}$ is given in Fig. 1 . The composite radio spectrum on 28 Oct. resembles the modality in Fig. 1, but cannot be put here because of the page limit. The radio emission on 26 started at about 6:13 UT with a group of fast type III bursts which extended over all of the radio frequency range at 6:15 UT. Meanwhile some DPS were found in the range of $25 \mathrm{MHz}-1.5 \mathrm{GHz}$. After 6:49 UT several interplanetary type II bursts were found which continued to about 9:12 UT. There were type IV bursts abroad in the global radio range lasting more than 2.5 hours. In summary, this event included globally complicated structures such as type IV bursts, type III bursts, type II bursts and DPS. For the event on 27 Oct., some fast type III bursts were found after 7:58 UT over almost the whole radio range. At 8:07 UT several DPS appeared first near $1.0 \mathrm{GHz}$, then they drifted to low-frequency nearly 20

$\dagger$ This work supported by NSFC grants $10225313 \& 10333030$ and MOST grant G2000078403. 

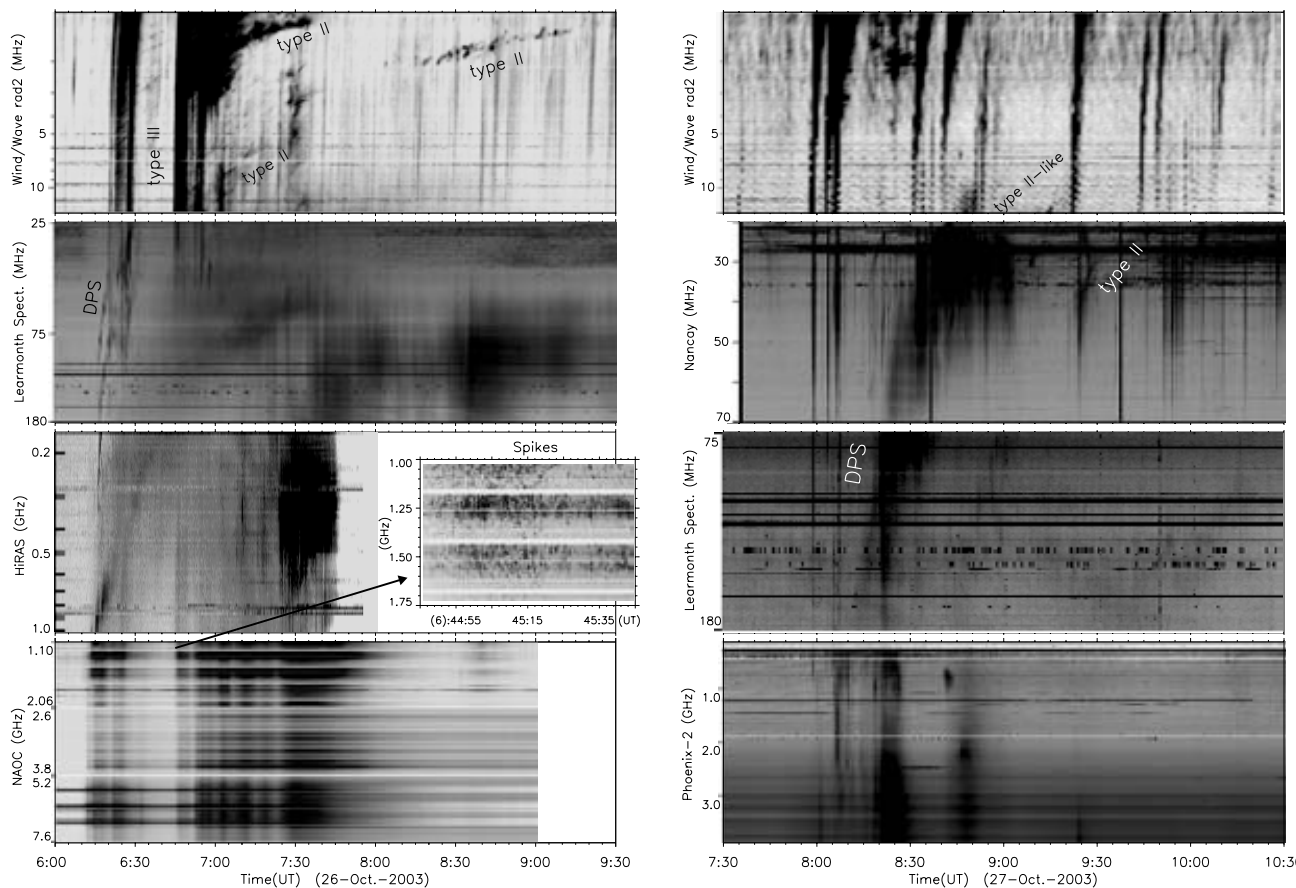

Figure 1. Composite radio spectra on 26 (a group of spikes in the inset) and 27 Oct., 2003

$\mathrm{MHz}$ until to 9:03 UT. Afterwards a type II burst was recorded by DAM/Nançay after 9:20 UT. Also in the range 10-14 MHz there seemed to be two slower drifting structures at about 8:45 UT and 9:15 UT (type II-like bursts). Then for the event on 28 Oct., the global radio spectrum was also very complicated including type IV bursts, type III bursts, type II-like bursts and DPS. Meanwhile using our millisecond data, many fine structures were also detected including narrow band drifting lines, zebra pattern, fiber bursts, patch and many spikes (an example showed in the inset of the left of Fig 1.).

Recently, a kind of slowly negatively drifting pulsation structures (DPS) was presented and interpreted as a signature of dynamic magnetic reconnection by Kliem et al., (2000) and Karlicky et al., (2001). The slow negative drift could caused by the upward motion of the plasmoid of the whole reconnection region to one of lower plasma density. DPS are closely associated with CME. It is possible that DPS in decimetric frequency band manifests the initial phase of the CME. In our events, DPS were found to be the typical signatures of those radio bursts associated with CMEs. Also the composite spectra indicate there were many complicated structures of radio bursts accompanying with the large CMEs including type II burst, type IV burst, type III burst and DPS, and many kinds of radio fine structures. Therefore it could be indicated that the strong solar activity associated with complicated magnetic structures and magnetic reconnection processes.

\section{References}

Delannèe, C., Delaboudiniére, J. -P \& Lamy, P. 2000, Astron. Astrophys. 355, 725

Karlický, M., Yan, Y., Fu, Q. et al. 2001, Astron. Astrophys. 369, 1104

Kliem, B., Karlický, M. \& Benz, A. O. 2000, Astron. Astrophys. 360, 715

St. Cyr, O. C., Burkepile, J. T., Hundhausen, A. J. et al. 1999, J. Geophys. Res. 104, 12493

Wang, S. J., Yan, Y., Zhao, R. et al. 2001, Solar Physics 204(1), 153 